Vol. 46 (1992) [81-90]

\title{
RIGHT ALTERNATIVE ALGEBRAS WITH COMMUTATORS IN A NUCLEUS
}

\author{
ErWin Kleinfeld and Harry F. Smith
}

\begin{abstract}
Let $A$ be a right alternative algebra, and $[A, A]$ be the linear span of all commutators in $A$. If $[A, A]$ is contained in the left nucleus of $A$, then left nilpotence implies nilpotence. If $[A, A]$ is contained in the right nucleus, then over a commutative-associative ring with $1 / 2$, right nilpotence implies nilpotence. If $[A, A]$ is contained in the alternative nucleus, then the following structure results hold: (1) If $A$ is prime with characteristic $\neq 2$, then $A$ is either alternative or strongly $(-1,1)$. (2) If $A$ is a finite-dimensional nil algebra, over a field of characteristic $\neq 2$, then $A$ is nilpotent. (3) Let the algebra $A$ be finite-dimensional over a field of characteristic $\neq 2,3$. If $A / K$ is separable, where $K$ is the nil radical of $A$, then $A$ has a Wedderburn decomposition
\end{abstract}

\section{INTRODUCTION}

Let $A$ be a nonassociative algebra. As is customary, for $x, y, z \in A$ we denote by $(x, y, z)$ the associator $(x, y, z)=(x y) z-x(y z)$ and by $[x, y]$ the commutator $[x, y]=x y-y x$. If the algebra $A$ satisfies the identity

$$
(y, x, x)=0
$$

then it is called right alternative. A right alternative algebra which also satisfies the identity $(x, x, y)=0$ is called alternative, and one which satisfies the identity $[[x, y], z]=0$ is called strongly $(-1,1)$.

In any nonassociative algebra $A$, the following are subalgebras:

$$
\begin{aligned}
& N_{\ell}=\{n \in A \mid(n, x, y)=0 \text { for all } x, y \in A\} \text { - left nucleus, } \\
& N_{\tau}=\{n \in A \mid(x, y, n)=0 \text { for all } x, y \in A\} \text { - right nucleus. }
\end{aligned}
$$

For $A$ a right alternative algebra with characteristic $\neq 2,3$,

$$
U=\{u \in A \mid[u, x]=0 \text { for all } x \in A\} \text { - commutative centre }
$$

Received 10 July 1991

Copyright Clearance Centre, Inc. Serial-fee code: 0004-9729/92 \$A2.00+0.00. 
is a subalgebra of $A$; and for characteristic $\neq 2$,

$$
N_{\beta}=\{v \in A \mid(x, x, v)=0 \text { for all } x \in A\} \text { - alternative nucleus }
$$

is a subalgebra with both $N_{r} \subseteq N_{\beta}$ and $U \subseteq N_{\beta}$.

For $A$ a nonassociative algebra, if for some positive integer $n$ every product of $n$ elements from $A$ is zero, no matter how the elements are associated, then $A$ is called nilpotent. Less restrictively, let $A_{[1]}=A$ and define inductively $A_{[k]}=A A_{[k-1]}$. If $A_{[n]}=0$ for some $n$, then $A$ is said to be left nilpotent. Analogously, setting $A^{[1]}=A$ and defining inductively $A^{[k]}=A^{[k-1]} A$, then $A$ is right nilpotent if $A^{[n]}=0$ for some $n$.

In Section 2 we consider left and right nilpotency in certain varieties of right alternative algebras. Let $[A, A]$ denote the linear span of all commutators in an algebra $A$. Then for $A$ a right alternative algebra with $[A, A] \subseteq N_{\ell}$, we show that for each natural number $n$ there exists a natural number $f(n)$ such that $A^{f(n)} \subseteq A_{[n]}$. In particular, if $A$ is left nilpotent, then $A$ is nilpotent. We next consider a right alternative algebra $A$, over a commutative-associative ring with $1 / 2$, such that $[A, A] \subseteq N_{r}$. We show that for such an algebra $A$ right nilpotence implies nilpotence. In particular, if such an $A$ satisfies the minimum condition on right ideals, then its quasi-regular radical $J(A)$ is nilpotent. We also note that existing examples $[2,11,16]$ can be used to show that in these indicated varieties there are no other implications between left or right nilpotence and nilpotence.

Let $A$ be right alternative algebra with characteristic $\neq 2$. It is known that if $[A, A] \subseteq N_{\beta}$, then $A$ is alternative if $A$ is either simple [19] or prime and finitelygenerated [9]. In Section 3 we first extend these results by showing that if $A$ is prime with $[A, A] \subseteq N_{\beta}$, then $A$ is either alternative or strongly $(-1,1)$. We then assume $A$ is a finite-dimensional right alternative algebra with $[A, A] \subseteq N_{\beta}$, and prove the following: (1) If $A$ is a nil algebra over a field of characteristic $\neq 2$, then $A$ is nilpotent. (2) (Wedderburn Decomposition) Let the algebra $A$ be over a field with characteristic $\neq 2,3$, and $A / K$ be separable, where $K$ is the nil radical of $A$. Then there exists a subalgebra $S$ of $A$ such that $A=S \oplus K$ (vector space direct sum). It is known that neither of these results holds for finite-dimensional right alternative algebras in general $[\mathbf{2}, \mathbf{2 0}]$.

Finally, we note that in addition to (1) we shall also make use of the following identities:

$$
\begin{aligned}
& (x, y, z)+(x, z, y)=0 \\
& {[x y, z]-x[y, z]-[x, z] y=(x, y, z)-(x, z, y)+(z, x, y)} \\
& (x y, z, w)+(x, y,[z, w])=x(y, z, w)+(x, z, w) y \\
& {[[x, y], z]+[[y, z], x]+[[z, x], y]=2\{(x, y, z)+(y, z, x)+(z, x, y)\}}
\end{aligned}
$$


Identity $\left(1^{\prime}\right)$ is just the linearised form of (1). A straightforward verification shows that (2) holds in any algebra. Identities (3) and (4) hold in any right alternative algebra with characteristic $\neq 2$, for example see $[19]$.

\section{NiLPOTENCY}

We first consider the variety of right alternative algebras which satisfy the identity $([x, y], z, w)=0$. As usual, for any algebra $A$ we denote by $L_{a}$ and $R_{a}$ the operators of left and right multiplication by $a \in A$. Using an argument analogous to that used by Slin'ko for $(-1,1)$ algebras [12], we prove:

Theorem 1. Let $A$ be a right alternative algebra such that $[A, A] \subseteq N_{\ell}$. For each natural number $n$ there exists a natural number $f(n)$ such that $A^{f(n)} \subseteq A_{[n]}$.

Proof: As noted in [16], if $I$ is an ideal in a right alternative algebra $A$, then $A I$ is also an ideal. In particular, $A_{[n]}$ is an ideal of $A$ for each $n$.

Our proof will be by induction on $n$. Since $A=A_{[1]}$ and $A^{2}=A_{[2]}$, we start with $f(1)=1$ and $f(2)=2$. Suppose then there exists a number $f(n-1) \geqslant 2$ such that $A^{f(n-1)} \subseteq A_{[n-1]}$. We first consider $A_{[n-1]} R_{x_{1}} \ldots R_{x_{k}}$, where $k \geqslant 3$. The identity $([x, y], z, w)=0$ written in operator form gives

$$
R_{y} R_{z} R_{w}=R_{y} R_{z w}+L_{y} R_{z} R_{w}-L_{y} R_{z w} .
$$

Using this to substitute for $R_{x_{1}} R_{x_{2}} R_{x_{3}}$, we see $A_{[n-1]} R_{x_{1}} R_{x_{2}} R_{x_{3}} \ldots R_{x_{k}} \subseteq$ $A_{[n-1]} R_{x_{1}} R_{x_{2} x_{3}} \ldots R_{x_{k}}+A_{[n-1]}\left(L_{x_{1}} R_{x_{2}} R_{x_{3}} \ldots R_{x_{k}}-L_{x_{1}} R_{x_{2} x_{3}} \ldots R_{x_{k}}\right)$. Thus, since $A_{[n-1]} L_{x_{1}} \subseteq A_{[n]}$ and $A_{[n]}$ is an ideal, we have $A_{[n-1]} R_{x_{1}} \ldots R_{x_{k}} \subseteq A_{[n-1]} R_{x_{1}} R_{x_{2} x_{3}} \ldots$ $R_{x_{k}}+A_{[n]}$ Applying this same argument to $A_{[n-1]} R_{x_{1}} R_{x_{2} x_{3}} \ldots R_{x_{k}}$, after $k-2$ such procedures we arrive at $A_{[n-1]} R_{x_{1}} \ldots R_{x_{k}} \subseteq A_{[n-1]} R_{x_{1}} R_{\left(\left(x_{2} x_{3}\right) \ldots\right) x_{k}}+A_{[n]}$. Now let $k-1=f(n-1)$. Then using $A_{[n-1]}$ is an ideal and our induction assumption, we see $\left.A_{[n-1]} R_{x_{1}} R_{\left(\left(x_{2} x_{3}\right) \ldots\right) x_{k}} \subseteq A_{[n-1}\right] R_{A^{f(n-1)}} \subseteq A_{[n-1]} R_{A_{[n-1]}} \subseteq A_{[n-1]} A_{[n-1]} \subseteq A_{[n]}$. Thus we have $A_{[n-1]} R_{x_{1}} \ldots R_{x_{f(n-1)+1}} \subseteq A_{[n]}$, and so it follows that

$$
A_{[n-1]} S_{1} \ldots S_{f(n-1)+1} \subseteq A_{[n]} \text {, where } S_{i} \text { is either } L_{x_{i}} \text { or } R_{x_{i}} \text {. }
$$

We now let $t>1$ be an integer such that $2^{t-1}<f(n-1)+1 \leqslant 2^{t}$. Then $A^{2^{t+f(n-1)+1}} \subseteq A^{2^{t+f(n-1)}} S_{1} \subseteq \ldots \quad \subseteq A^{2^{t}} S_{1} \ldots S_{f(n-1)+1} \subseteq$ $A^{f(n-1)} S_{1} \ldots S_{f(n-1)+1} \subseteq A_{[n-1]} S_{1} \ldots S_{f(n-1)+1} \subseteq A_{[n]}$, using our induction assumption and (*). Thus it suffices to take $f(n)=2^{t+f(n-1)+1}$, which completes our induction and the proof of the theorem.

Corollary. Let $A$ be a right alternative algebra such that $[A, A] \subseteq N_{\ell}$. If $A$ is left nilpotent, then $A$ is nilpotent.

In [2] Dorofeev constructed an example of a finite-dimensional right alternative algebra that is right nilpotent but not nilpotent. This algebra $A$ has basis $\{a, b, c, d, e\}$, 
with the nonzero products of basis elements being $a b=-b a=a e=-e a=d b=-b d=$ $-c, a c=d, b c=e$. A straightforward computation shows that $[A, A]$ is contained in the subspace with basis $\{c, d, e\}$, and then that $[A, A] \subseteq N_{\ell}$. We also note that the subspaces with bases $\{a, c, d, e\}$ and $\{b, c, d, e\}$ are nilpotent ideals whose sum is $A$. Thus it follows that the locally nilpotent radical doesn't exist in the variety of right alternative algebras satisfying $([x, y], z, w)=0$.

We next consider nilpotency in the variety of right alternative algebras which satisfy the identity $(x, y,[z, w])=0$.

THEOREM 2. Let $A$ be a right alternative algebra, over a commutative-associative ring with $1 / 2$, such that $[A, A] \subseteq N_{r}$. If $A$ is right nilpotent, then $A$ is nilpotent.

Proof: First, for any nonassociative algebra $A$, let $A^{(1)}=A$ and define inductively $A^{(n)}=\left(A^{(n-1)}\right)^{2}$. Then if $A^{(m)}=0$, with $m$ the least such integer, the algebra $A$ is called solvable of index $m$. Now it is immediate that any right nilpotent algebra is solvable, and so to prove the theorem we induct on the index of solvability of $A$. For a start, it is clear $A$ is nilpotent when $A=A^{(1)}=0$ or $A^{2}=A^{(2)}=0$. Thus by induction we can assume $A^{2}$ is nilpotent, since $A^{2}$ is a right nilpotent right alternative algebra which satisfies $(x, y,[z, w])=0$ and has solvable index one less than that of $A$. In particular, let $\left(A^{2}\right)^{n}=0$.

Now from the proof of Theorem 1 in [7], $\bar{N}_{r}=\left\{n \in N_{r} \mid n A \subseteq N_{r}\right\}$ is an ideal of $A$ such that $[[A, A], A] \subseteq \bar{N}_{r}$. Thus $A / \bar{N}_{r}$ is a right nilpotent strongly $(-1,1)$ algebra, over a commutative-associative ring with $1 / 2$, and so by Theorem 5 in [10] $A / \bar{N}_{r}$ is nilpotent. In particular, we must have $(A) L_{x_{1}} \ldots L_{x_{m}} \subseteq \bar{N}_{r}$ for some integer $m>0$. Also, using that $\bar{N}_{r}$ is an ideal contained in $N_{r}$, for $2 n$ factors of $A$ we have $A\left(A\left(\ldots A\left(A \bar{N}_{r}\right)\right)\right)=A^{2}\left(A\left(\ldots A\left(A \bar{N}_{r}\right)\right)\right)=\ldots=A^{2}\left(A^{2}\left(\ldots A^{2}\left(A^{2} \bar{N}_{r}\right)\right)\right)=$ $\left(A^{2}\right)^{2}\left(A^{2}\left(\ldots A^{2}\left(A^{2} \bar{N}_{r}\right)\right)\right)=\ldots=\left(\left(\left(\left(A^{2}\right)^{2} A^{2}\right) A^{2} \ldots\right) A^{2}\right) \bar{N}_{r} \subseteq\left(A^{2}\right)^{n} \bar{N}_{r}=0$ that is $\left(\bar{N}_{r}\right) L_{y_{1}} \ldots L_{y_{2 n}}=0$. Thus it now follows that $(A) L_{x_{1}} \ldots L_{x_{m}} L_{y_{1}} \ldots L_{y_{2 n}} \subseteq$ $\left(\bar{N}_{r}\right) L_{y_{1}} \ldots L_{y_{2 n}}=0$, and so $A$ is left nilpotent. But by Lemma 1 in [16], a right alternative algebra that is both left and right nilpotent is nilpotent. This completes our induction, and so proves the theorem.

Corollary. Let $A$ be a right alternative algebra, over a commutative-associative ring containing $1 / 2$, such that $[A, A] \subseteq N_{r}$. If $A$ satisfies the minimum condition on right ideals, then the quasi-regular radical $\mathrm{J}(A)$ of $A$ is nilpotent.

Proof: By [15] $\mathbf{J}(A)$ is right nilpotent, and so by Theorem $2 \mathbf{J}(A)$ is in fact nilpotent.

In [11] Pchelincev constructed an example of a right nilpotent right alternative algebra $A$ that is not nilpotent. We note that a straightforward verification shows $[A, A] \subseteq N_{\beta}$, so Theorem 2 cannot be extended to the variety of right alternative 
algebras satisfying $(x, x,[y, z])=0$. Also, in [16] Slin'ko constructed an example of a left nilpotent right alternative algebra $A$ that is not nilpotent. This example has the property $A A^{2}=0$, and so obviously satisfies the identity $(x, y,[z, w])=0$.

\section{Alternative nucleus}

In this section we consider the variety of right alternative algebras which satisfy the identity $(x, x,[y, z])=0$.

Proposition 1. Let $A$ be a right alternative algebra with characteristic $\neq 2$. If $[A, A] \subseteq N_{\beta}$, then $\bar{N}_{\beta}=\left\{v \in N_{\beta} \mid v A \subseteq N_{\beta}\right\}$ is an ideal of $A$ such that $\left[N_{\beta}, A\right] \subseteq$ $\bar{N}_{\beta}$.

Proof: By Theorem 2 in [19], $\bar{N}_{\beta}$ is an ideal of $A$. Let $\nu \in N_{\beta}$ and $y, z \in A$. Using (2) and $\left(1^{\prime}\right)$, we see

$$
\begin{aligned}
{[v, z] y } & =[v y, z]-v[y, z]-(v, y, z)+(v, z, y)-(z, v, y) \\
& =[v y, z]-v[y, z]+2(v, z, y)+(z, y, v) .
\end{aligned}
$$

Now $[A, A] \subseteq N_{\beta}$ by assumption, and $N_{\beta}$ is a subalgebra of $A$ by Lemma 1 in [19]. Also, $\left(N_{\beta}, A, A\right) \subseteq N_{\beta}$ by the Corollary to Lemma 6 in [19]; and $\left(A, A, N_{\beta}\right) \subseteq N_{\beta}$ by Lemma 3.1 in [9]. Thus it follows $[v, z] y \in N_{\beta}$, that is, $\left[N_{\beta}, A\right] \subseteq \bar{N}_{\beta}$, which completes the proof.

As usual, an algebra $A$ is prime if $B C=0$ for ideals $B$ and $C$ of $A$ implies either $B=0$ or $C=0$.

THEOREM 3. Let $A$ be a prime right alternative algebra with characteristic $\neq 2$. If $[A, A] \subseteq N_{\beta}$, then $A$ is either alternative or strongly $(-1,1)$.

Proof: Let $M$ be the submodule of $A$ generated by all associators of the form $(x, x, y)$. By Lemma 11 in [19], $M+M A$ is an ideal of $A$ such that $(M+M A) \bar{N}_{\beta}=0$. Since $A$ is prime, either $M+M A=0$, so $A$ is alternative; or by Proposition 1, $[[A, A], A] \subseteq\left[N_{\beta}, A\right] \subseteq \bar{N}_{\beta}=0$, so $A$ is strongly $(-1,1)$.

Corollary. Let $A$ be a prime right alternative algebra with characteristic $\neq$ 2, 3. If $[A, A] \subseteq N_{\beta}$, then $A$ is alternative if $A$ satisfies any of the following conditions:

(i) $A$ is without nonzero locally nilpotent ideals,

(ii) $A$ is finitely-generated,

(iii) $A$ has an idempotent $e \neq 0,1$,

(iv) $A$ satisfies the minimum condition on right or left ideals.

Proof: Let $A$ be a strongly $(-1,1)$ algebra. Then $A$ is associative under condition (i) by Corollary 2 to Theorem 3 in [19]. If $A$ is prime, then $A$ is associative 
under condition (ii) by Theorem 5 in [4], and under condition (iii) by Theorem 2 in [18]. Under condition (iv), the locally nilpotent radical of $A$ is nilpotent by Theorem 3 in [12]. Since if $I$ is an ideal of $A$ so is $I^{k}$, this means that if $A$ is prime, then condition (iv) implies condition (i), that is, $A$ is associative.

Theorem 4. Let $A$ be a finite-dimensional right alternative nil algebra over a field of characteristic $\neq 2$. If $[A, A] \subseteq N_{\beta}$, then $A$ is nilpotent.

Proof: We first note that, over a field of characteristic $\neq 2$, any finitedimensional right alternative nil algebra is right nilpotent, for example [15]. Our proof of the theorem will be by induction on the dimension of $A$, with $\operatorname{dim}(A)=1$ being immediate. Now by Proposition 1 we have $[[A, A], A] \subseteq \bar{N}_{\beta}$. Thus if $\bar{N}_{\beta}=0$, then $A$ is a strongly $(-1,1)$ nil algebra, and so $A$ is nilpotent by Theorem 4 in $[3]$. We can therefore assume $\bar{N}_{\beta} \neq 0$, and then let $I$ be a minimal nonzero ideal of $A$ contained in $\bar{N}_{\beta}$. As noted in the proof of Theorem $1, A I$ is also an ideal of $A$; and so by the minimality of $I$ we must have either $A I=0$ or $A I=I$.

Suppose first that it is the case that $A I=0$. Now by induction the algebra $A / I$ is nilpotent, since $I \neq 0$ implies $\operatorname{dim}(A / I)<\operatorname{dim}(A)$. Thus $A^{n} \subseteq I$ for some integer $n$, whence $A_{[n+1]}=A A_{[n]} \subseteq A A^{n} \subseteq A I=0$. This shows the right alternative algebra $A$ is both left and right nilpotent, and so in this cas $A$ is nilpotent by Lemma 1 in [16].

We suppose next that it's the case $A I=I$. By $\left(1^{\prime}\right)$ we have $\left(A^{2} I\right) A \subseteq\left(A^{2} A\right) I+$ $A^{2}(I A)+A^{2}(A I) \subseteq A^{2} I$. Also, since $I \subseteq N_{\beta}$ implies

$$
(x, y, m)+(y, x, m)=0 \text { for all } x, y \in A \text { and } m \in I,
$$

we see $A\left(A^{2} I\right) \subseteq A^{2}(A I)+\left(A A^{2}\right) I+\left(A^{2} A\right) I \subseteq A^{2} I$. Thus $A^{2} I$ is an ideal of $A$, and so by the minimality of $I$ we have either $A^{2} I=0$ or $A^{2} I=I$. We suppose first that $A^{2} I=I$. Since $A$ is right nilpotent, we know $A^{2} \neq A$. Thus by induction the ideal $A^{2}$ is nilpotent, say $\left(A^{2}\right)^{k}=0$. Then for $k$ factors of $A^{2}$, since $A^{2} I=I$ we have $I=A^{2}\left(A^{2}\left(\ldots\left(A^{2}\left(A^{2} I\right)\right)\right)\right) \subseteq\left(A^{2}\right)^{k}=0$, which is a contradiction. Suppose next that $A^{2} I=0$. We let $\left\{x_{1}, \ldots, x_{s}\right\}$ be a basis for $A$ and consider a product of the form $x_{i_{s+1}}\left(x_{i_{s}}\left(\ldots\left(x_{i_{2}}\left(x_{i_{1}} I\right)\right)\right)\right)$, where the $s+1$ factors $x_{i_{j}}$ are any elements from this basis. Now from $\left({ }^{* *}\right)$ and $A^{2} I=0$, we see $x(y I)=-y(x I)$ for any $x, y \in A$. Then since $I$ is an ideal contained in $N_{\beta}$, and since some basis element $x_{i}$ must appear as a factor twice in the indicated product, we see $x_{i_{1+1}}\left(x_{i_{1}}\left(\ldots\left(x_{i_{2}}\left(x_{i_{1}} I\right)\right)\right)\right)=$ $\pm x_{j}\left(x_{j}\left(\ldots\left(x_{i_{k}} I\right)\right)\right) \subseteq x_{j}^{2} I \subseteq A^{2} I=0$. Thus for $s+1$ factors of $A$, since $A I=I$ it now follows that $I=A(A(\ldots(A(A I))))=0$, which again is a contradiction. This then shows the case $A I=I$ is impossible, which completes our induction and the proof of the theorem.

We next let $e \neq 0,1$ be an idempotent in a right alternative algebra $A$ with characteristic $\neq 2$. With respect to $e$, one has the Albert decomposition $A=A_{1} \oplus$ 
$H_{1} \oplus H_{0} \oplus A_{0}$ (module direct sum), where $A_{i}=\{x \in A \mid e x=i x=x e\}, H_{1} \oplus H_{0}=$ $\{x \in A \mid e x+x e=x\}, B_{1} e \subseteq A_{1}$, and $e H_{0} \subseteq A_{0}$ [1]. If $(e, e, A)=0$, then this Albert decomposition can be refined to the Peirce decomposition $A=A_{11} \oplus A_{10} \oplus A_{01} \oplus A_{00}$ (module direct sum), where $A_{i j}=\{x \in A \mid e x=i x, x e=j x\}$ for $i, j=0,1$. In this latter case, one also has the following multiplication table for the submodules $A_{i j}$ [5]:

\begin{tabular}{c||c|c|c|c|} 
& $A_{11}$ & $A_{10}$ & $A_{01}$ & $A_{00}$ \\
\hline \hline$A_{11}$ & $A_{11}+A_{01}$ & $A_{10}$ & $A_{10}$ & 0 \\
\hline$A_{10}$ & 0 & $A_{11}+A_{01}$ & $A_{11}$ & $A_{10}$ \\
\hline$A_{01}$ & $A_{01}$ & $A_{00}$ & $A_{10}+A_{00}$ & 0 \\
\hline$A_{00}$ & 0 & $A_{01}$ & $A_{01}$ & $A_{10}+A_{00}$ \\
\hline
\end{tabular}

Proposition 2. Let $A$ be a right alternative algebra, with characteristic $\neq 2$, such that $[A, A] \subseteq N_{\beta}$. If $e \neq 0,1$ is an idempotent in $A$, then $A$ permits a Peirce decomposition with respect to $e$, and the multiplication table is as follows:

\begin{tabular}{c||c|c|c|c|} 
& $A_{11}$ & $A_{10}$ & $A_{01}$ & $A_{00}$ \\
\hline \hline$A_{11}$ & $A_{11}+A_{01}$ & $A_{10}$ & 0 & 0 \\
\hline$A_{10}$ & 0 & $A_{01}$ & $A_{11}$ & $A_{10}$ \\
\hline$A_{01}$ & $A_{01}$ & $A_{00}$ & $A_{10}$ & 0 \\
\hline$A_{00}$ & 0 & 0 & $A_{01}$ & $A_{10}+A_{00}$ \\
\hline
\end{tabular}

Also, if $x_{i j}$ denotes a generic element of $A_{i j}$, then $x_{i j}^{2}=0$ for $i \neq j$.

Proof: First, setting $x=y=e$ in (3) and using $[A, A] \subseteq N_{\beta}$, we see $e(e, z, w)+(e, z, w) e=\left(e^{2}, z, w\right)+(e, e,[z, w])=(e, z, w)$, that is $(e, A, A) \subseteq$ $H_{1} \oplus H_{0}$. In particular, this means $\left(e, e, H_{i}\right) \subseteq A_{i} \cap\left(H_{1} \oplus H_{0}\right)=0$. Thus $(e, e, A)=\left(e, e, A_{1}+H_{1}+H_{0}+A_{0}\right)=\left(e, e, H_{1}\right)+\left(e, e, H_{0}\right)=0$, and so $A$ permits a Peirce decomposition with respect to $e$.

Next, since $[A, A] \subseteq N_{\beta}$, we have $(i-j) x_{i j}=\left[e, x_{i j}\right] \in N_{\beta}$ for $i \neq j$, that is $\left(y, z, x_{i j}\right)=-\left(z, y, x_{i j}\right)$. Using this and the indicated multiplication table for a Peirce decomposition in any right alternative algebra, we can now compute as follows. First $(j-i) x_{i j} y_{i j}=\left(x_{i j}, e, y_{i j}\right)=-\left(e, x_{i j}, y_{i j}\right)=-i x_{i j} y_{i j}+e\left(x_{i j} y_{i j}\right)$, whence $e\left(x_{i j} y_{i j}\right)=$ $j x_{i j} y_{i j}$. Thus $A_{i j} A_{i j} \subseteq A_{j i}$. Next $(i-j) x_{i i} y_{j i}=\left(x_{i i}, e, y_{j i}\right)=-\left(e, x_{i i}, y_{j i}\right)=0$, since $e\left(x_{i i} y_{j i}\right)=i x_{i i} y_{j i}$. Thus $A_{i i} A_{j i}=0$. This then establishes the multiplication table as stated in the proposition, and from it we see that also $(i-j) x_{i j}^{2}=\left(e, x_{i j}, x_{i j}\right)=0$ by (1).

COROLlary. If $A$ is a right alternative algebra, with characteristic $\neq 2$, such that $[A, A] \subseteq N_{\beta}$, then any idempotent in $A$ is in $N_{\beta}$.

Proof: Since it is clear we can assume the idempotent $e \neq 0,1$, we let $x=$ $x_{11}+x_{10}+x_{01}+x_{00}$. Now from just the definition of $A_{i j}$, we see $\left(x_{i j}, e, x_{j k}\right)=0$. 
Also, from the multiplication table in Proposition 2 and the fact that $x_{i j}^{2}=0$ for $i \neq j$, we see $\left(x_{i i}, e, x_{j i}\right)=\left(x_{i i}, e, x_{j j}\right)=\left(x_{i j}, e, x_{i i}\right)=\left(x_{i j}, e, x_{i j}\right)=0$. Thus by $\left(1^{\prime}\right)$ $(x, x, e)=-(x, e, x)=-\left(x_{11}+x_{10}+x_{01}+x_{00}, e, x_{11}+x_{10}+x_{01}+x_{00}\right)=0$ for all $x \in A$, which proves the corollary.

We note that the multiplication table in Proposition 2 cannot be reduced further to that for an alternative algebra. For let $A$ be the finite-dimensional algebra with basis $\left\{1, e, z_{10}, x_{00}, y_{00}\right\}$, where 1 is a unity, $e^{2}=e$, and the only other nonzero products of basis elements are $e z_{10}=x_{00} y_{00}=-y_{00} x_{00}=z_{10}$. A straightforward verification shows that over any field $A$ is a right alternative algebra. Also, the subspace $[A, A]$ has basis $\left\{z_{10}\right\}$, whence it follows directly that $[A, A] \subseteq\left(N_{\ell} \cap N_{r}\right) \subseteq N_{\beta}$. However, $A_{00}^{2} \nsubseteq A_{00}$ for the idempotent $e$, and $A_{11}^{2} \nsubseteq A_{11}$ for the idempotent $1-e$.

Theorem 5. (Wedderburn Decomposition). Let $A$ be a finite-dimensional right alternative algebra, over a field $F$ of characteristic $\neq 2,3$, with $[A, A] \subseteq N_{\beta}$. If $A / K$ is separable, where $K$ is the nil radical of $A$, then there exists a subalgebra $S$ of $A$ such that $A=S \oplus K$ (vector space direct sum).

Proof: The proof is by induction on the dimension of $A$, with the initial case $\operatorname{dim}(A)=1$ being immediate. Then as in [8], by induction one can assume the nil radical $K$ of $A$ does not properly contain any nonzero ideals of $A$. Let $\langle$ Alt $\rangle$ denote the ideal of $A$ generated by all associators of the form $(x, x, y)$. Then by $[14,15]$ we have $\langle$ Alt $\rangle \subseteq K$. Now if $\langle$ Alt $\rangle=0$, then the algebra $A$ is alternative; and so $A$ has a Wedderburn decomposition by [13]. Thus we can assume $K=\langle$ Alt $\rangle$.

We next let $S(x y, x, y)=(x y, x, y)+(x, y, x y)+(y, x y, x)$. Now since the algebra $A /\langle$ Alt $\rangle$ is alternative, by the well-known Artin's theorem we must have $S(x y, x, y) \in\langle$ Alt $\rangle$. Also, by Proposition 1 the ideal $\bar{N}_{\beta}$ contains $[[x y, x], y]+$ $[[x, y], x y]+[[y, x y], x]$. Thus by identity (4) we see $2 S(x y, x, y) \in\langle$ Alt $\rangle \cap \bar{N}_{\beta}$. This means that if $\langle$ Alt $\rangle \cap \bar{N}_{\beta}=0$, then the algebra $A$ must satisfy the identity $S(x y, x, y)=0$; and in this case $A$ has a Wedderburn decomposition by Theorem 5 in [17]. Thus we can now assume $\langle$ Alt $\rangle=K \subseteq \bar{N}_{\beta}$. In particular, by Lemma 11 in [19] we now have $\langle\text { Alt }\rangle^{2} \subseteq\langle$ Alt $\rangle \bar{N}_{\beta}=0$, and so as in [8] one can assume the base field $F$ to be algebraically closed.

Now since $K=\langle$ Alt $\rangle$, by $[15]$ we know $A /\langle$ Alt $\rangle \simeq B_{1} \oplus \ldots \oplus B_{t}$, where each minimal ideal $B_{i}$ is either an associative matrix algebra over a division ring or a Cayley-Dickson algebra. Since $\langle$ Alt $\rangle \subseteq \bar{N}_{\beta}$, we can thus take the ideal $\bar{N}_{\beta} /\langle$ Alt $\rangle \simeq B_{k+1} \oplus \ldots \oplus B_{t}$ (or 0 ), whence $A / \bar{N}_{\beta} \simeq(A /\langle$ Alt $\rangle) /\left(\bar{N}_{\beta} /\langle\right.$ Alt $\left.\rangle\right) \simeq B_{1} \oplus \ldots \oplus B_{k}$ (where $k=t$ if $\bar{N}_{\beta}=\langle$ Alt $\rangle$ ). Now by Proposition 1 we have $[[A, A], A] \subseteq \bar{N}_{\beta}$, so $A / \bar{N}_{\beta}$ is a strongly $(-1,1)$ algebra. Thus for $1 \leqslant i \leqslant k$ each $B_{i}$ is a simple strongly $(-1,1)$ algebra with idempotent. Since characteristic $F \neq 2,3$, by [6] this means each of these $B_{i}$ 's is a field. But the field $F$ is algebraically closed, so for $1 \leqslant i \leqslant k$ we must in fact have 
$B_{i} \simeq F\left[u_{i}\right]$, where $\left[u_{i}\right]=u_{i}+\langle$ Alt $\rangle$ is idempotent.

Now $\left[u_{i}^{m}\right]=\left[u_{i}\right]^{m}=\left[u_{i}\right]$, so $u_{i}$ cannot be nilpotent. Thus the finite-dimensional associative subalgebra generated by $u_{i}$ in $A$ must contain an idempotent $e_{i}=f\left(u_{i}\right)$, where $f(x)$ is some polynomial over $F$. Then $\left[e_{i}\right]=\left[f\left(u_{i}\right)\right]=\alpha\left[u_{i}\right]$, where $\alpha=f(1) \in$ $F$; so $\alpha\left[u_{i}\right]=\left[e_{i}\right]=\left[e_{i}\right]^{2}=\alpha^{2}\left[u_{i}\right]^{2}=\alpha^{2}\left[u_{i}\right]$. Now the idempotent $e_{i}$ cannot be in the nil radical $K=\langle$ Alt $\rangle$, so $\alpha\left[u_{i}\right] \neq 0$, that is $\alpha \neq 0$. Thus $\alpha=1$, and so each $F\left[u_{i}\right]=F\left[e_{i}\right]$ where $e_{i}$ is an idempotent in $A$. In particular, by the Corollary to Proposition 2, each $e_{i} \in N_{\beta}$.

We now take a basis for $K=\langle$ Alt $\rangle \subseteq \bar{N}_{\beta}$, and extend this to a basis $\left\{x_{1}, \ldots, x_{s}\right\}$ for $\bar{N}_{\beta}$. Then $\left\{x_{1}, \ldots, x_{s}, e_{1}, \ldots, e_{k}\right\} \subseteq N_{\beta}$ will be a basis for $A$. But this means the algebra $A$ is alternative, and so as noted earlier $A$ has a Wedderburn decomposition by [13]. This then completes our induction, and with it the proof of the theorem.

\section{REFERENCES}

[1] A.A. Albert, 'The structure of right alternative algebras', Ann. of Math. 59 (1954), 408-417.

[2] G.V. Dorofeev, 'The nilpotency of right alternative rings', (Russian), Algebra i Logika 9 (1970), 302-305.

[3] I.R. Hentzel, '(-1, 1) rings', Proc. Amer. Math. Soc. 22 (1969), 367-374.

[4] I.R. Hentzel, 'Nil semi-simple $(-1,1)$ rings', J. Algebra 22 (1972), 442-450.

[5] M.M. Humm, 'On a class of right alternative rings without nilpotent ideals', J. Algebra 5 (1967), 164-174.

[6] E. Kleinfeld, 'On a class of right alternative rings', Math. $Z .87$ (1965), 12-16.

[7] E. Kleinfeld and H.F. Smith, 'On simple rings with commutators in the left nucleus', Comm. Algebra 19 (1991), 1593-1601.

[8] I.M. Miheev, "The theorem of Wedderburn on the splitting of the radical for a $(-1,1)$ algebra', (Russian), Algebra i Logika 12 (1973), 298-304.

[9] $\mathrm{Ng}$ Seong Nam, 'Alternative nucleus of right alternative algebras', Southeast Asian Bull. Math. 10 (1986), 149-154.

[10] S.V. Pchelincev, 'Nilpotency of the associator ideal of a free finitely generated $(-1,1)$ ring', (Russian), Algebra i Logika 14 (1975), 543-572.

[11] S.V. Pchelincev, 'The locally nilpotent radical in certain classes of right alternative rings', (Russian), Sibirsk. Mat. Zh. 17 (1976), 340-360.

[12] R.E. Roomel'di, 'Nilpotency of ideals in a $(-1,1)$ ring with minimum condition', (Russian), Algebra i Logiki 12 (1973), 333-348.

[13] R.D. Schafer, 'The Wedderburn principal theorem for alternative algebras', Bull. Amer. Math. Soc. 55 (1949), 604-614.

[14] V.G. Skosyrskī̄, 'Right alternative algebras', (Russian), Algebra i Logika 23 (1984), 185-192. 
[15] V.G. Skosyrskiī, 'Right alternative algebras with minimality condition for right ideals', (Russian), Algebra i Logika 24 (1985), 205-210.

[16] A.M. Slin'ko, 'The equivalence of certain nilpotencies of right alternative rings', (Russian), Algebra i Logika 9 (1970), 342-348.

[17] H.F. Smith, 'Finite-dimensional locally $(-1,1)$ algebras', Comm. Algebra 7 (1979), 177-191.

[18] N.J. Sterling, 'Prime $(-1,1)$ rings with idempotent', Proc. Amer. Math. Soc. 18 (1967), 902-909.

[19] A. Thedy, 'Right alternative rings', J. Algebra 37 (1975), 1-43.

[20] A. Thedy, 'Right alternative algebras and Wedderburn principal theorem', Proc. Amer. Math. Soc. 72 (1978), 427-435.

Division of Mathematical Sciences

University of Iowa

Iowa City, IA 52242

United States of America
Department of Mathematics

Statistics and Computing Science

University of New England

Armidale NSW 2351 